\title{
Short-term Tests of Potassium dldD Vector Magnetometer
}

\author{
Eugen VUJIĆ \\ Faculty of Geodesy, University of Zagreb, Zagreb, Croatia \\ e-mail: eugvujic@gfz.hr
}

\begin{abstract}
In this paper the results of short-term tests of a suspended Potassium dIdD vector magnetometer are presented. The instrument was installed on a stable pillar during May 2012 near Milton (Ontario, Canada). The aim was to investigate primarily the bias current stability and the noise level of the instrument (for measured components less than $10 \mathrm{pT}$ in a root-mean-square sense). A brief description of the measurement principles and a review of formulae used for field component calculations are given. The errors of components, which arise from assumptions on dIdD orientation as well as from the total field time variations during a measurement cycle, are estimated and discussed, both for real and simulated data. Finally, the accuracy of the instrument was estimated, and the maximal theoretical accuracies of components were better than $60 \mathrm{pT}$ at mid-latitudes.
\end{abstract}

Key words: Potassium dIdD vector magnetometer, magnetometer calibration, error calculation.

\section{INTRODUCTION}

The temporal variations of the geomagnetic field are continuously recorded at geomagnetic observatories and periodically at variometer stations. The most common instruments are triaxial fluxgate instruments, which are relative instruments and have to be calibrated periodically by measurements of

Ownership: Institute of Geophysics, Polish Academy of Sciences;

(C) 2015 Vujić. This is an open access article distributed under the Creative Commons

Attribution-NonCommercial-NoDerivs license,

http://creativecommons.org/licenses/by-nc-nd/3.0/. 
the absolute instruments, such as D/I Fluxgate and (Overhauser effect) proton magnetometers. However, in remote areas and over the oceans there is a need for automation of absolute measurements (Newitt et al. 1996, Auster H.U. et al. 2007 and references therein).

During the recent twelve years, substantial efforts were made in developing the automated absolute instruments. The automation of D/I Fluxgate was presented in Van Loo and Rasson (2007). The method of performing the absolute measurements without the theodolite, and using a scalar magnetometer and a three axis fluxgate magnetometer rotating about two defined axes is presented in Auster and Auster (2003), and further developed in Auster H.U. et al. (2007). The basic idea is that the calibration of the magnetometer and the determination of the field in direction of the rotation axis can be done with the same procedure. The calibration principle by using a scalar magnetometer is given, e.g., in Pang et al. (2013). A processor controlled proton magnetometer design and its application for an automated absolute measurement is presented in Auster V. et al. (2007).

There is also a class of magnetometers which measure field components by a scalar sensor equipped with coil systems (Jankowski and Sucksdorff 1996, Auster H.U. et al. 2007 and references therein). In this class are the dIdD vector magnetometers. The deflected Inclination deflected Declination (dIdD) method of measuring was first proposed by Alldredge (1960), using a proton precession sensor, and by Alldredge and Saldukas (1964), using an optically pumped rubidium sensor. The development of suspended Overhauser effect dIdD, which uses the Overhauser effect magnetometer sensor inside the two orthogonal bias coils, was briefly described in Hegymegi et al. (2004). The temperature characteristics of this instrument were given in Csontos et al. (2007).

The first three-component magnetometer using a scalar potassium sensor was presented in Alexandrov et al. (2004). During recent years, the Potassium dIdD was developed, with a potassium magnetometer sensor inside the bias coils. The first example of usage of this instrument was given in Hrvoic et al. (2009), and it was used as the variometer during very sensitive $(1 \mathrm{fT} / \mathrm{m})$ SuperGradiometer installation in Oaxaca State (Mexico). The dIdD vector magnetometers are actually semi-absolute instruments, i.e., once the calibration parameters are known, the absolute measurements can be derived (Heilig 2007). These instruments are also automatically operated, so after their initial calibration they represent the automated absolute magnetometers. Still, under temperature and humidity uncontrolled conditions, it is advisable to make the occasional re-calibrations (Csontos et al. 2012).

The aims of this work are listed hereafter. The prime objectives of mesurements performed with Potassium dIdD, which are presented here, were to test the bias coils fields' stability and instrumental noise levels. Fur- 
ther, the geomagnetic components deviations due to the dIdD orientation errors are estimated, both for real and simulated data, which were derived for quiet and disturbed conditions at locations of four observatories in western hemisphere, in equatorial, mid-latitude, auroral, and polar regions, respectively. Also, the influence of assumptions on calibration of real data is presented. The errors of components due to the total field time variations during measurements were estimated, both for real and simulated data (at four observatories), for different sampling rates and geomagnetic activity levels. Moreover, the instrumental accuracy of measured components was obtained, and also the maximal theoretical accuracies as functions of the bias fields at locations of four observatories. Finally, the estimations of the calibration parameters' accuracies, which are necessary for the instrumental long-term stability up to $5 \mathrm{nT}$, were derived.

\section{A BRIEF REVIEW OF THE USED MAGNETOMETER}

\subsection{Theory of instrument operation}

Alkali vapor optically pumped magnetometers use gaseous alkali metals, such as potassium. A vapour cell containing gaseous metal is exposed by light of a specific wavelength. Let us consider, e.g., three energy levels of atoms (two ground states, 1 and 2, with energy levels $E_{1}$ and $E_{2}$, and excited state 3 , with energy level $E_{3}$, with assumption that $E_{1}<E_{2}<<E_{3}$ ). The frequency of light is specifically selected and circularly polarized, to excite atoms from level 2 to 3 . The atoms at level 3 spontaneously decay to both energy levels 1 and 2. Eventually the level 1 is fully populated and level 2 is depleted. Further, RF (radio frequency) power is applied to the cell to move atoms from level 1 back to level 2. The frequency of the RF field required to repopulate level 2 varies with the local magnetic field over a sensor, and is actually the Larmor frequency. The light intensity becomes modulated by the $\mathrm{RF}$, and by detecting its modulation and measuring the frequency one can obtain a value of the local magnetic field (Alexandrov and Bonch-Bruevich 1992, Hrvoic and Newitt 2011). The block diagram of potassium magnetometer and its explanation is given, e.g., in Alexandrov and BonchBruevich (1992).

The suspended Potassium dIdD (P-dIdD) consists of a potassium magnetometer sensor centered inside two orthogonal spherical coil systems (Fig. 1a, attained at http://www.gemsys.ca). Coils are eventually aligned to be approximately perpendicular to the local geomagnetic field direction in the horizontal and geomagnetic meridian planes, respectively. High level of orthogonality of the two bias coils can be achieved experimentally by monitoring their mutual induction. Each measurement cycle consists of five sequences: bias currents in both directions are applied to both coil system, the 


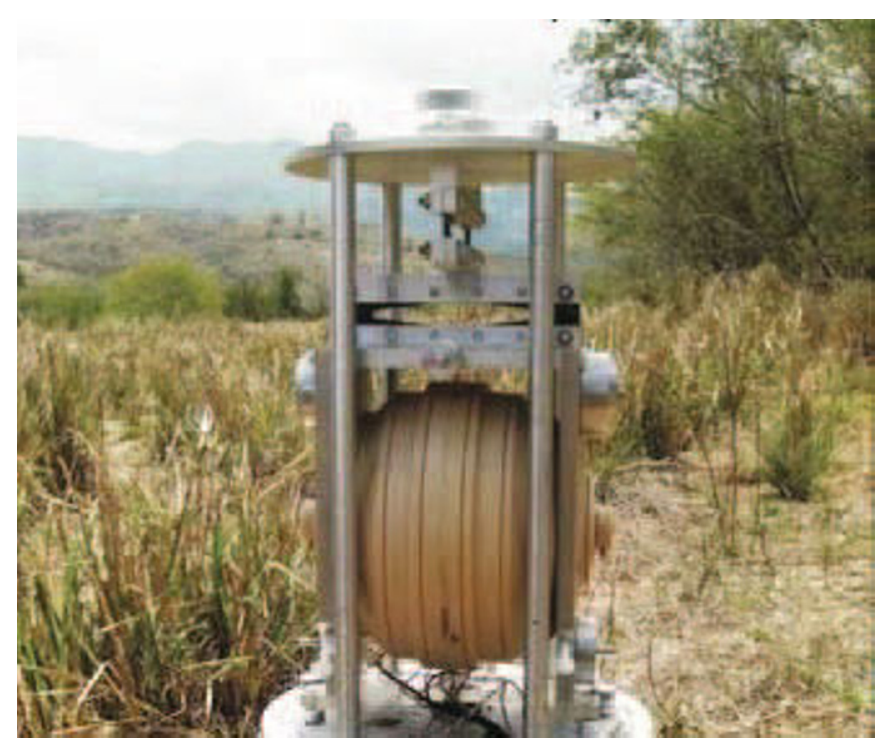

Fig. 1a. The suspended P-dIdD on a pillar. The outer spherical bias coils (D-coils) can be seen.

four biased total fields along with the unbiased total field are measured in a cycle (Schott et al. 2001, Heilig 2007, Hrvoic and Newitt 2011). The mechanical design of suspended P-dIdD is the same as for suspended Overhauser effect dIdD (Hegymegi et al. 2004), with potassium magnetometer sensor inside the bias coils instead of Overhauser effect proton magnetometer sensor.

A duration of one sequence (a sequence time) is five times shorter than sampling interval (a cycle time). These sequences are denoted as $D_{p}, D_{m}, F$, $I_{p}$, and $I_{m}$ (Fig. $1 \mathrm{~b}$ ). $D_{p}$ is a local field over sensor when D-coils bias field is towards the magnetic east, $D_{m}$ when this bias field is in opposite direction, $F$ is a local field without any bias, $I_{p}$ is a field when I-coils bias field is towards the Earth's surface, and $I_{m}$ is a field over sensor when this bias field is in opposite direction (Heilig 2007, Hrvoic and Newitt 2011). Switching from one bias to another requires a delay for transients to die out, but the time required is so short that either instrument can be considered approximately continuous (this time interval is about $80 \mu$ s for D-coils, and about $60 \mu$ s for I-coils). As with most optically pumped magnetometers, the P-dIdD sensor should be properly oriented with respect to the direction of magnetic field. The ideal angle between sensor axis and magnetic field is $45^{\circ} \pm 35^{\circ}$ or $135^{\circ} \pm 35^{\circ}$. Beyond these limits, the Larmor signal will be weakened and eventually faded away; these dependencies are known from Dehmelt (1957), and Bell and Bloom (1957) experiments. 


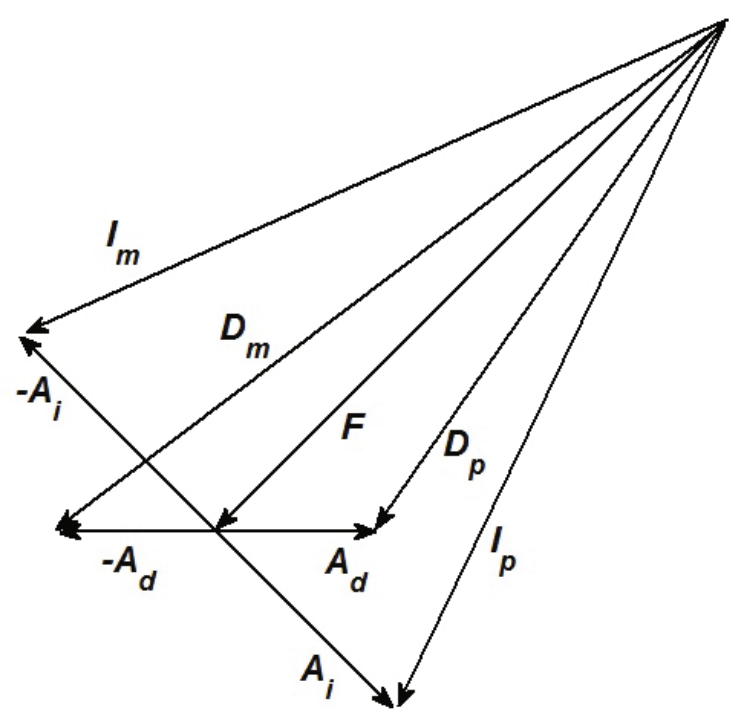

Fig. 1b. Measurement principle of P-dIdD: $D_{p}, D_{m}, F$ (the local total field), $I_{p}$ and $I_{m}$ are measured components; $A_{d}$ and $A_{i}$ are D-coils and I-coils bias fields, respectively.

\subsection{Calculation of the geomagnetic field components}

In this analysis two coordinate reference frames will be used. The first one is orthogonal right-handed $X Y Z$-frame, with positive $X$-axis in direction of geographic north, positive $Y$-axis in direction of geographic east, and $Z$-axis in direction of local vertical and is positive downward (Campbell 2003). The second one is SDI-frame (dIdD reference frame, not necessarily orthogonal), and it is always defined by the orientation of the bias coils axes. The two axes (D and I) are parallel to D- and I-coils axes, respectively, and the third axis (S) is defined to be perpendicular to both coils axes. It is defined that the dIdD is aligned when the D-axis is horizontal and D- and I-axes are orthogonal, and oriented when both axes are perpendicular to the local geomagnetic field vector. In case when the bias coils are not oriented but are aligned, $D_{0}$ will be an angle between D-coil plane and a local geographic meridian, while $I_{0}$ will be an angle between I-coil plane and horizontal plane (Schott and Leroy 2001, Heilig 2007). The D- and I-coil bias magnetic fields are $A_{d}=\left[\left(D_{p}{ }^{2}+D_{m}{ }^{2}-2 F^{2}\right) / 2\right]^{0.5}$ and $A_{i}=\left[\left(I_{p}{ }^{2}+I_{m}{ }^{2}-2 F^{2}\right) / 2\right]^{0.5}$, respectively. The total field components in SDI-frame are (Schott et al. 2001): $B_{D}=\left(D_{p}{ }^{2}-D_{m}{ }^{2}\right) / 4 A_{d}, B_{I}=\left(I_{p}{ }^{2}-I_{m}{ }^{2}\right) / 4 A_{i}$ and $B_{S}=\left(F^{2}-B_{D}{ }^{2}-B_{I}{ }^{2}\right)^{0.5}$.

Let us consider a real case when $\mathrm{dIdD}$ is not aligned and oriented. The formalism given in Heilig (2007) is used. The orthogonality error between $\mathrm{D}$ - and I-axes is $\varepsilon_{O}$, and this error can be removed by using the transfor- 
mations of total field components from non-orthogonal to orthogonal SDIframe (the one where SDI-axes form a right-handed coordinate system, denoted by '), which are given by:

$$
\begin{aligned}
& \left(\begin{array}{l}
B_{D}^{\prime} \\
B_{I}^{\prime}
\end{array}\right)=\left(\begin{array}{cc}
1 / \cos \varepsilon_{O} & -\tan \varepsilon_{O} \\
0 & 1
\end{array}\right)\left(\begin{array}{l}
B_{D} \\
B_{I}
\end{array}\right), \\
& B_{S}^{\prime}=\left[F^{2}-\left(B_{D}^{2}+B_{I}^{2}-2 B_{D} B_{I} \sin \varepsilon_{O}\right) / \cos ^{2} \varepsilon_{O}\right]^{0.5} .
\end{aligned}
$$

The next step is to set a D-coil axis horizontal. This can be achieved if the orthogonal SDI-frame is rotated by angle $\theta$ about its S-axis in clockwise direction, when looking towards the origin. The matrix of that rotation is $\boldsymbol{\Theta}$. Now one has perfectly aligned, but not oriented dIdD. One has to make two additional rotations to obtain $X Y Z$-frame. The first is a rotation of SDI-frame about D-axis by angle $I_{0}$, in a counterclockwise direction when looking towards the origin. This makes S-axis horizontal, I-axis vertical, the corresponding rotation matrix is $\mathbf{I}$. The last rotation is about I-axis by angle $D_{0}$ in a clockwise direction when looking towards the origin, and a rotation matrix is D. Finally one has that SDI-frame coincides with $X Y Z$-frame. Now the field conponents in $X Y Z$-frame can be expressed with those in SDI-frame (Heilig 2007):

$$
\left(\begin{array}{c}
X \\
Y \\
Z
\end{array}\right)=\mathbf{D} \mathbf{I} \Theta\left(\begin{array}{c}
B_{S}^{\prime} \\
B_{D}^{\prime} \\
B_{I}^{\prime}
\end{array}\right) .
$$

\section{MEASUREMENTS AND dIdD CALIBRATION}

The suspended P-dIdD was installed at an artificial noise-free location (Fig. 2) near town Milton (Ontario, Canada), on a nonmagnetic stable pillar, where the horizontal and vertical total field gradients are in the range of 1-2 nT/m, and covered by a shelter. The differences between measured biased fields after dIdD installation were $D_{p}-D_{m}<2 \mathrm{nT}$ and $I_{p}-I_{m}<2 \mathrm{nT}$. The instrument operated in the period 11-15 May 2012, and powered from AC power supply. The instrument has $0.1 \mathrm{pT}$ resolution and was sampling at $1 \mathrm{~Hz}$ sampling rate (i.e., the duration of one measurement sequence was $0.2 \mathrm{~s}$ ). Since the total field modulus during measurement sequence was not constant, the stage duration must be much longer than the atomic transition relaxation times, which are of the order of $30 \mathrm{~ns}$ (Happer 1972), to avoid transient effects. The aim of these measurements was to test dIdD data aquisition software, the bias field current source stability, and instrumental noise level. 


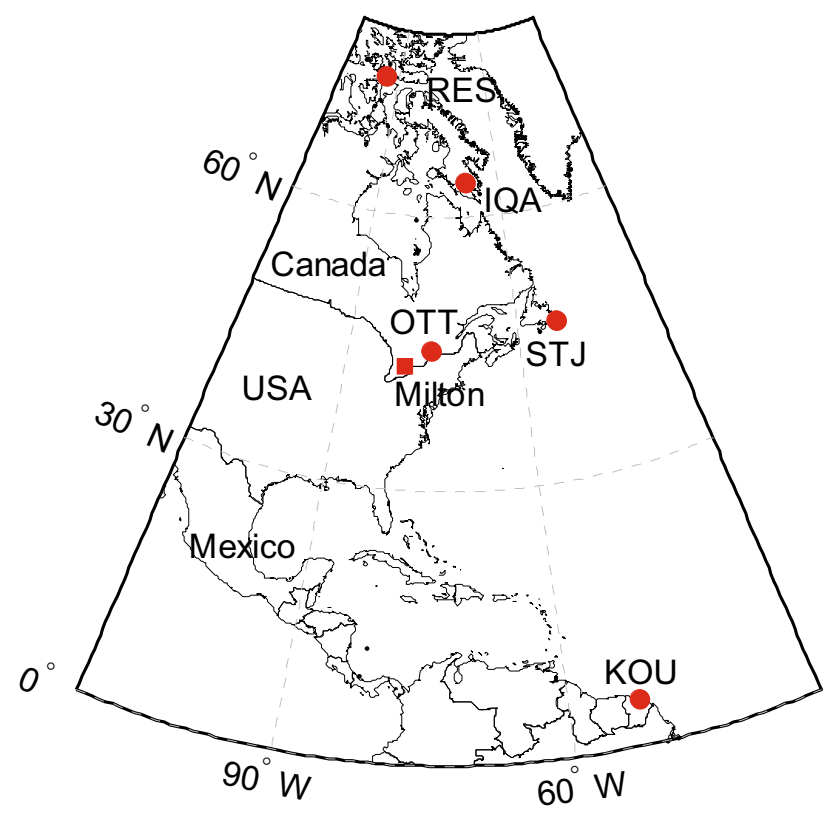

Fig. 2. The location of Milton (square), Ottawa observatory (OTT), and the observatories (RES, IQA, STJ, and KOU) used for the estimation of virtual dIdD errors and accuracies.

The absolute measurements of declination, inclination (both with $\mathrm{D} / \mathrm{I}$ Fluxgate), and total field intensity (with Overhauser effect proton magnetometer) were performed on 11 May, after the dIdD setup (installed on 10 May 2012), on a tripod some $20 \mathrm{~m}$ away from dIdD pillar. For $D$ and $I$ measurements a null-method was used (Newitt et al. 1996). Two high precision GPS receivers in differential mode were used for the determination of the azimuth of the reference mark (Gu et al. 2006). One GPS was used as a reference mark, about $120 \mathrm{~m}$ away from the DI tripod (due to practical reasons), and a second GPS receiver was set on the tripod. The reference azimuth error was estimated to be less than 8 ".

After dIdD setup and absolute measurements, one can make a dIdD vector calibration (Heilig 2007), i.e., to derive the angles $D_{0}, I_{0}, \theta$, and $\varepsilon_{O}$ (see Section 2.2). The calibration was derived under the assumption that the time variations of geomagnetic field components are the same at $\mathrm{dIdD}$ and absolute instrument, and that the differences in $X, Y$, and $Z$ components between these two sites are known (non zero-gradient field). These site differences can be estimated by absolute measurements taken on both sites. The total field difference between dIdD and absolute instruments' site was $3.5 \mathrm{nT}$. The following condition for the geomagnetic field components was applied: 


$$
\sum_{i}\left(E_{i(\mathrm{abs})}-E_{i(\mathrm{ddd})}\right)=\sum_{i} \Delta E_{i},
$$

where $E=X, Y$ or $Z$, subscript "abs" is for absolute measurements and dIdD for P-dIdD measurements (their one-minute averages), and $\Delta E$ is a difference between absolute and dIdD sites. Considering summation index $i$, there are seven options: if $i=3$, then one has summation $X+Y+Z$; if $i=2$, one may have $X+Y, X+Z$ or $Y+Z$; and if $i=1$, one will have only condition for $X, Y$ or $Z$. It was found numerically for what values of parameters $D_{0}, I_{0}$, $\theta$, and $\varepsilon_{O}$ the condition 3 is fulfilled, within $0.01 \mathrm{nT}$, for all of the seven options simultaneously. The estimated uncertainties are $\left(D_{0}, I_{0}, \theta\right.$, and $\left.\varepsilon_{O}\right)$ : $\pm 0.0008^{\circ}, \pm 0.0003^{\circ}, \pm 0.024^{\circ}$, and $\pm 0.0168^{\circ}$. One-minute averages were determined by using the Gaussian low-pass filter (St-Louis 2004).

\section{DATA ANALYSIS AND DISCUSSION}

\subsection{The bias fields stability}

Generally, for calculation of field components it is assumed that the bias currents are stable during one measurement cycle, i.e., one can presume that $\boldsymbol{A}_{\boldsymbol{d}}$ and $-\boldsymbol{A}_{\boldsymbol{d}}$ are antiparallel and have the same amplitude, and also for $\pm \boldsymbol{A}_{\boldsymbol{i}}$. This is not true in practice since current source could produce fluctuations within one cycle. One has to consider the so-called hypothesis of symmetrical polarization (Marsal et al. 2007), i.e., to verify an assumption about the same amplitude of bias fields during one cycle, respectively. The aim was to search for anomalous and asymmetrical polarizations, and a formalism proposed in Marsal et al. (2007) for both bias fields was applied and adjusted for the used cycle time.

First one has to find anomalous polarizations, and that means to find the sudden spikes in bias field time series. After that, one has to find asymmetrical polarization in each cycle, which provides information about the symmetrical hypothesis of bias field. The spikes in both polarisations (in a given cycle) indicate an erroneous value. In this case, percentage of the erroneous values (i.e., seconds) during five days was $0.0028 \%$ ( 2.4 per day), with $5 \mathrm{~mA}$ bias coils' current. These stability tests' results are about twice better than those when noncalibrated P-dIdD was operated in 2011 for two months on the same pillar, with $6 \mathrm{~mA}$ bias coils' current. Also, the standard deviations of first differences for $A_{d}$ and $A_{i}$ were 3 and $1 \mathrm{pT}$, respectively.

Figure 3 shows the hourly mean air temperature (attained at http:// climate.weatheroffice.gc.ca/climateData) from Weather Station Guelph Turfgrass $\left(\varphi=43.55^{\circ} \mathrm{N}, \lambda=80.05^{\circ} \mathrm{W}\right)$, which is some $18 \mathrm{~km} \mathrm{SW}$ away from measurement site, and the hourly mean values of D- and I-coils bias fields during five days. In both cases, the negative linear correlations were derived, and they are very high, -0.9315 and -0.9335 for D- and I-coils bias 

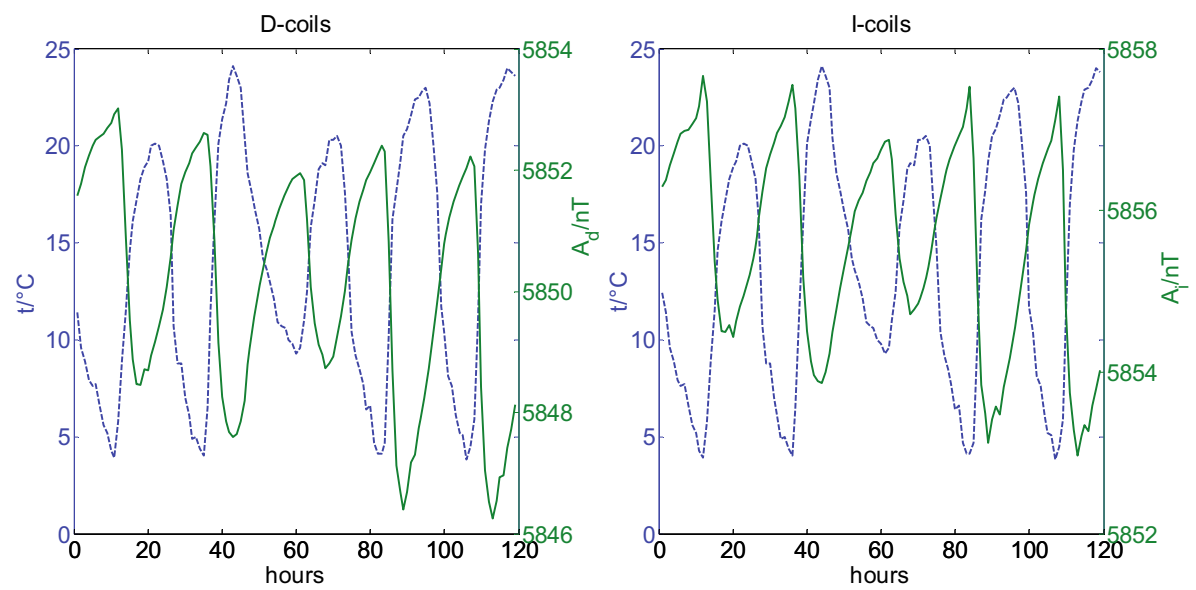

Fig. 3. Hourly mean air temperature at Weather Station Guelph Turfgrass (blue) and hourly mean values of D- and I-coils bias fields (green). D-coils bias field is in the interval from 01:00 on 11 May to 00:00 on 16 May 2012, and I-coils bias field is from 00:00 on 11 May to 23:00 on 15 May 2012. Temperature is in the interval from 00:00 on 11 May to 23:00 on 15 May 2012 (all in UTC).

field, respectively. The clear temperature dependence of the bias fields was also reported by Marsal et al. (2007), but for more controlled observatory conditions, for different bias coil design and for a dIdD that uses proton magnetometer sensor. They have obtained that for temperature variations of $1{ }^{\circ} \mathrm{C}$ the bias field variations were about $1 \mathrm{nT}$, although for differently designed dIdD. In this case, there were about $0.33 \mathrm{nT}$ (outer, D-coils) and about $0.23 \mathrm{nT}$ (inner, I-coils) changes for $1{ }^{\circ} \mathrm{C}$ external temperature variations. These "thermal coefficients" are only their effective values, because dependences of the bias fields on ambient temperature are not linear, and the corresponding temperature differences at Milton and Guelph station are (probably) not the same.

\subsection{Components deviations due to the dIdD orientation errors}

After dIdD calibration it is possible to estimate the errors arising from the the instrument misorientation, i.e., due to different values of $D_{0}, I_{0}, \theta$, and $\varepsilon_{O}$ taken to calculate the geomagnetic components. The results for maximal errors are given in Table 1, and they are based on all one-minute averages. The first row gives the errors of components when there are two cases: in the first one the values for $D_{0}, I_{0}, \theta$, and $\varepsilon_{O}$ derived as a results of instrument calibration were used, and in the second the values for $D_{0}$ and $I_{0}$ (i.e., $D_{0}^{\prime}$ and $I_{0}^{\prime}$ ) were used, and they originated from assumption that during calibration there is $\theta=0$ and $\varepsilon_{O}=0$. In the second row are the errors when there are also 
two cases: in the first one the values for $D_{0}, I_{0}, \theta$, and $\varepsilon_{O}$ obtained from instrument calibration were used, and in the second one $D_{0}, I_{0}, \theta$, and $\varepsilon_{O}$ (i.e., $D_{0 a}, I_{0 a}, \theta_{a}$, and $\varepsilon_{O a}$ ) were derived under the assumption that $X, Y$, and $Z$ components are the same at absolute instrument and dIdD sites during calibration (zero-gradient field).

Table 1

The maximal absolute errors of calculated geomagnetic components, but with different assumptions about the dIdD orientation (see text for details)

\begin{tabular}{|c|c|c|c|}
\hline P-dIdD errors & $\begin{array}{c}\operatorname{Max}|\Delta X| \\
{[\mathrm{nT}]}\end{array}$ & $\begin{array}{c}\operatorname{Max}|\Delta Y| \\
{[\mathrm{nT}]}\end{array}$ & $\begin{array}{c}\operatorname{Max}|\Delta Z| \\
{[\mathrm{nT}]}\end{array}$ \\
\hline $\begin{array}{l}D_{0} I_{0} \theta \\
D_{0}^{\prime} I_{0}^{\prime} 0\end{array}$ & 0.4 & 0.4 & 0.1 \\
\hline $\begin{array}{l}D_{0} I_{0} \theta \quad \varepsilon_{O} \\
D_{0 a} I_{0 a} \theta_{a} \varepsilon_{O a}\end{array}$ & 0.5 & 0.6 & 0.2 \\
\hline
\end{tabular}

In Figure 4 there are shown the maximal absolute errors, when only one calibration parameter has been changed by $-0.1^{\circ}$ to $0.1^{\circ}$, and the others were constant. In a case of $X$ component, $\Delta I_{0}$ has the largest and $\Delta \varepsilon_{O}$ the smallest influence, and the errors due to $\Delta I_{0}$ and $\Delta D_{0}$ are much larger than due to $\Delta \varepsilon_{O}$ and $\Delta \theta$. Considering $Y$ component errors, $\Delta D_{0}$ has the largest and $\Delta \theta$ the smallest influence, and the errors due to $\Delta D_{0}$ and $\Delta I_{0}$ are much larger than due to $\Delta \varepsilon_{O}$ and $\Delta \theta$. Finally, in the case of $Z$ component, $\Delta I_{0}$ has much larger influence than the other three parameters (their errors are less than $0.04 \mathrm{nT}$ ).
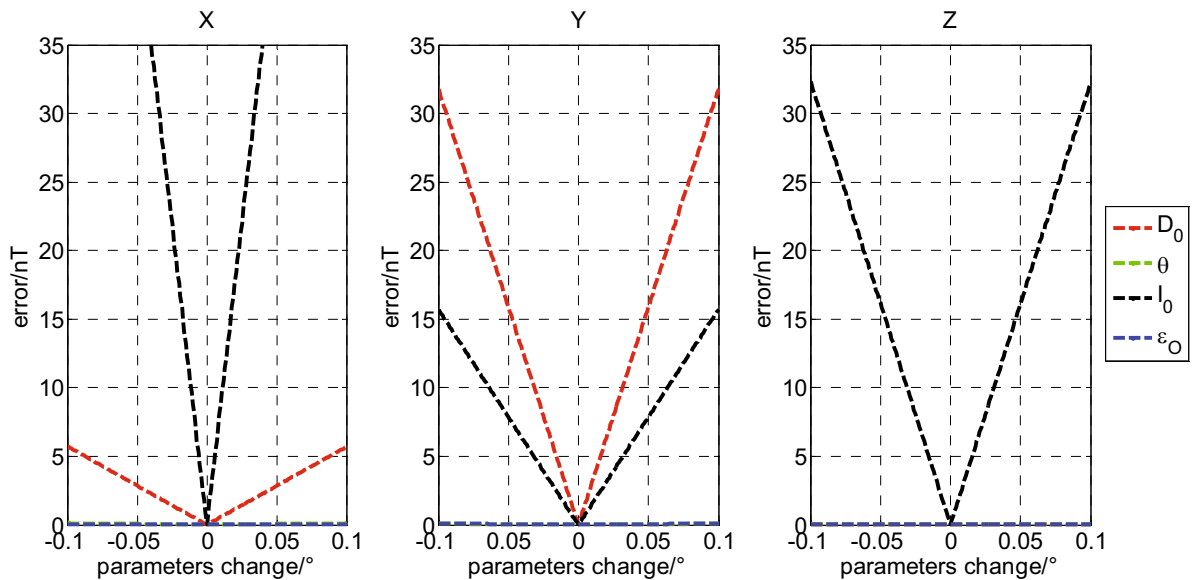

Fig. 4. The maximal absolute errors of $X, Y$, and $Z$ components when only one calibration parameter has been changed by $-0.1^{\circ}$ to $0.1^{\circ}$, and the others were constant. 
It is also possible to estimate these errors by putting a virtual dIdD in some observatory, since there the variations of $X, Y$, and $Z$ are known. This has been done for the following observatories (Fig. 2): Resolute (RES, polar cap region), Iqaluit (IQA, auroral oval region), St John's (STJ, mid-latitude region), and Kourou (KOU, equatorial region), during very disturbed days (severe geomagnetic storms from 29 to 31 October 2003) and very quiet geomagnetic conditions, respectively. The quiet daily variations were derived from SQ1 Model (Campbell 2003) for 30 October 2003. The dIdD parameters were taken under circumstances that $\mathrm{dIdD}$ is approximately oriented to the estimated quiet-night (QN) field, but with some realistic $\theta$ and $\varepsilon_{O}$ values, i.e., $D_{0}=D_{\mathrm{QN}}, I_{0}=I_{\mathrm{QN}}, \theta=0.5^{\circ}$, and $\varepsilon_{O}=0.1^{\circ}$. The following procedure was used: from corrections and rotations given in Section 2.2 it is possible to calculate bias fields in $X Y Z$-frame, and then using $X, Y, Z$, and $F$ one can calculate the angles between bias fields and total field vectors, respectively. After that, one can obtain $D_{p}, D_{m}, F, I_{p}$, and $I_{m}$.

Table 2

The maximal absolute errors of $X, Y$, and $Z$ components (one-minute values) when only one calibration parameter has been changed by $-0.1^{\circ}$ to $0.1^{\circ}$, and the others were constant

\begin{tabular}{|c|c|l|r|l|l|l|r|l|l|}
\hline \multirow{2}{*}{$\begin{array}{c}\text { Errors } \\
{[\mathrm{nT}]}\end{array}$} & \multicolumn{5}{c|}{ Quiet } & \multicolumn{4}{c|}{ Disturbed } \\
\cline { 2 - 9 } & $D_{0}$ & \multicolumn{1}{c|}{$I_{0}$} & \multicolumn{1}{c|}{$\theta$} & \multicolumn{1}{c|}{$\varepsilon_{O}$} & $D_{0}$ & $I_{0}$ & \multicolumn{1}{c|}{$\theta$} & $\varepsilon_{O}$ \\
\hline \multirow{3}{*}{ RES } & $X$ & 1.7 & 85.6 & 0.02 & 0.02 & 4.4 & 87.1 & 2.7 & 1.3 \\
& $Y$ & 2.7 & 54.1 & 0.03 & 0.03 & 3.8 & 55.0 & 3.9 & 2.1 \\
& $Z$ & 0.0005 & 3.3 & 0.001 & 0.0005 & 0.0005 & 4.5 & 0.1 & 0.09 \\
\hline \multirow{4}{*}{ IQA } & $X$ & 8.4 & 83.1 & 0.02 & 0.02 & 13.6 & 84.8 & 5.0 & 3.3 \\
& $Y$ & 13.0 & 53.8 & 0.03 & 0.03 & 14.4 & 54.9 & 8.0 & 5.1 \\
& $Z$ & 0.0005 & 15.5 & 0.003 & 0.0005 & 0.0005 & 16.4 & 1.1 & 0.3 \\
\hline \multirow{4}{*}{ STJ } & $X$ & 12.0 & 78.6 & 0.03 & 0.007 & 13.6 & 79.5 & 1.4 & 0.7 \\
& $Y$ & 31.7 & 29.8 & 0.02 & 0.02 & 33.4 & 30.1 & 1.9 & 1.8 \\
& $Z$ & 0.0005 & 33.9 & 0.01 & 0.0006 & 0.0005 & 35.9 & 0.6 & 0.02 \\
\hline \multirow{3}{*}{ KOU } & $X$ & 14.8 & 16.1 & 0.01 & 0.01 & 15.1 & 16.3 & 0.08 & 0.1 \\
& $Y$ & 46.7 & 5.1 & 0.03 & 0.03 & 46.9 & 5.2 & 0.5 & 0.4 \\
& $Z$ & 0.00005 & 49.0 & 0.02 & 0.0003 & 0.00005 & 49.2 & 0.3 & 0.004 \\
\hline
\end{tabular}

Note: The errors are estimated for solar quiet variation and severe geomagnetic storm, respectively, for a virtual dIdD in four observatories: RES, IQA, OTT, and KOU.

In Table 2 are maximal absolute errors (in nT) of $X, Y$, and $Z$ components (one-minute values) when only one calibration parameter has been changed by $-0.1^{\circ}$ to $0.1^{\circ}$, and the others were constant. It can be noticed that the errors due to $D_{0}$ and $I_{0}$ changes are higher for disturbed conditions, but not so 
relatively drastically as in the cases of $\theta$ and $\varepsilon_{O}$ changes, especially for IQA. For RES and IQA the errors are largest for $I_{0}$ changes, and smallest for $\varepsilon_{O}$ changes, but for RES during very disturbed conditions the errors in $X$ and $Y$, respectively, become comparable in the cases of $D_{0}$ and $\theta$ changes. The result for STJ shows a similar manner as that previously derived for Milton. For KOU observatory, in the cases of $X$ and $Y$ the errors due to $I_{0}$ and $D_{0}$ changes are much larger than those due to $\varepsilon_{O}$ and $\theta$ changes; they are slightly larger for $I_{0}$ changes in $X$, but for $Y$ component $D_{0}$ has a largest influence; in the case of $Z, I_{0}$ has much larger influence than the other three parameters. The parameter $D_{0}$ has negligible influence on $Z$; on $Y$ it has the largest influence, increasing from polar toward equatorial regions, and also for $X$ component. The parameter $I_{0}$ has an increasing influence on $X$ and $Y$ (higher for $X$ ) from equatorial toward polar regions, and increasing on $Z$ from polar toward equatorial regions.

\subsection{Errors due to time variations of the total field}

The total field vector has its time variations during one measurement cycle (Schott et al. 2001, Marsal et al. 2007), and this fact was neglected in Section 2.2. The components $D_{p}, D_{m}, F, I_{p}$, and $I_{m}$ are measured at the time instants $t_{1}, t_{2}, t_{3}, t_{4}$, and $t_{5}$, respectively. The idea is that all measurements have to be reduced to the time instant $t_{3}$. The assignments $D_{p}\left(t_{1}\right)=D_{p 1}$, $F\left(t_{3}\right)=F_{3}, I_{m}\left(t_{5}\right)=I_{m 5}$ are used. In this approach, the only approximation is that the amplitudes of $A_{d}$ and $A_{i}$ are constant over a single measurement cycle, i.e., that both polarizations are symmetrical, and it is assumed that the bias fields are homogenous over the sensor size. We have $\boldsymbol{D}_{p 3}=\boldsymbol{A}_{\boldsymbol{d}}+\boldsymbol{F}_{\mathbf{3}}=$ $A_{d}+F_{1}+\Delta F_{13}=D_{p 1}+\Delta F_{13}, \quad D_{m 3}=D_{m 2}+\Delta F_{23}, \quad I_{p 3}=I_{p 4}-\Delta F_{34} \quad$ and $\boldsymbol{I}_{\boldsymbol{m} \mathbf{3}}=\boldsymbol{I}_{\boldsymbol{m} \mathbf{5}}-\Delta \boldsymbol{F}_{\mathbf{3 5}}$, where $\Delta \boldsymbol{F}_{j k}$ denotes the total field changes frome instant $t_{j}$ to instant $t_{k}$. From these equations one can derive that $D_{p 3}, D_{m 3}, I_{p 3}$, and $I_{m 3}$ are functions of $D_{p 1}, D_{m 2}, I_{p 4}, I_{m 5}, \boldsymbol{F}_{\mathbf{3}}, \Delta \boldsymbol{F}_{\mathrm{ij}}, \boldsymbol{A}_{\boldsymbol{d}}$, and $\boldsymbol{A}_{\boldsymbol{i}}$ (Schott et al. 2001), respectively, e.g., $D_{p 3}=\left[D_{p 1}^{2}+2 \Delta \boldsymbol{F}_{\mathbf{1 3}} \cdot\left(\boldsymbol{A}_{\boldsymbol{d}}+\boldsymbol{F}_{\mathbf{3}}\right)-\left|\Delta \boldsymbol{F}_{\mathbf{1 3}}\right|^{2}\right]^{0.5}$ and $I_{m 3}=\left[I_{m 5}^{2}-\right.$ $\left.2 \Delta \boldsymbol{F}_{\mathbf{3 5}} \cdot\left(-\boldsymbol{A}_{\boldsymbol{i}}+\boldsymbol{F}_{\mathbf{3}}\right)-\left|\Delta \boldsymbol{F}_{\mathbf{3 5}}\right|^{2}\right]^{0.5}$.

These equations for $D_{p 3}, D_{m 3}, I_{p 3}$, and $I_{m 3}$ can be inserted into equations for $B_{D 3}, B_{I 3}, B_{S 3}, A_{d}$, and $A_{i}$ (see Section 2.2), to obtain a square nonlinear system of equations with the unknowns $\left(B_{D 3}, B_{I 3}, B_{S 3}, A_{d}, A_{i}\right)$, and solved numerically for each measurement cycle. In above equations $\Delta \boldsymbol{F}_{j k}$ have to be known from nearby triaxial variometer (Schott et al. 2001), which has to operate on a sampling rate higher or equal than dIdD sequence time. Since both conditions were not satisfied in the case of a Milton data, this method was tested on a virtual dIdD placed inside observatories RES, IQA, STJ, and KOU. 
Using the procedure from Section 4.2 one can derive $D_{p 1}, D_{m 2}, F_{3}, I_{p 4}$, and $I_{m 5}$ from $X, Y$, and $Z$, with the minimal sequence time $(\tau)$ of $1 \mathrm{~s}$, i.e., cycle time of $5 \mathrm{~s}$. This analysis was used in three cases: using the one-second variations registered at Milton, using a one-second observatories data on quiet day (6 December 2012) and on disturbed days (geomagnetic storm on 23/24 April 2012), respectively; the geomagnetic activity attained by using Ottawa Observatory $K$ index (http://geomag.nrcan.gc.ca). Also, the highest global activity indices on 23 April 2012 were $K p=6^{-}, A p=26 \mathrm{nT}$ and $D s t=-95 \mathrm{nT}$, and on 24 April 2012 they were $K p=7^{-}, A p=41 \mathrm{nT}$ and $D s t=-108 \mathrm{nT}$ ( $K p$ and $A p$ attained at http://www.gfz-potsdam.de; Dst attained at http://swdcwww.kugi.kyoto-u.ac.jp).

In Table 3a there are displayed the estimated maximal absolute errors of one-second Milton data, due to the differences of $D_{p 1}, D_{m 2}, I_{p 4}$, and $I_{m 5}$, and corresponding values reduced to $t_{3}$, respectively. The reduced values were estimated from measured one ( $\tau=0.2 \mathrm{~s}$ ) by adding: (a) constant values with random sign $(+\Delta$ or $-\Delta)$; and (b) random values with given amplitude $( \pm \Delta)$. The errors are largest for $Y$ and smallest for $Z$ component, and increase linearly with amplitude of differences; they are larger for case (a), and the presented values are averages over random 2000 cases, respectively.

In sequel, Table $3 \mathrm{~b}$ shows the mean absolute errors of geomagnetic components for observatories RES, IQA, STJ, and KOU, during quiet and disturbed conditions, respectively. These errors are larger during disturbed conditions, and then are largest in $Y$ and smallest in $Z$ component for RES, IQA and STJ, while for KOU are smallest in $X$, and about the same in $Y$ and $Z$. Also, they increase with sequence time, but it is not so obvious for KOU. These errors are largest for IQA and smallest for KOU during disturbed conditions. During quiet conditions, the errors are largest for STJ (possibly due

Table $3 \mathrm{a}$

The estimated $X, Y$, and $Z$ maximal absolute errors of Milton data due to the differences of $D_{p 1}, D_{m 2}, I_{p 4}$, and $I_{m 5}$,

and corresponding values reduced to $t_{3}$, respectively

\begin{tabular}{|l|c|c|l|c|c|l|}
\hline Errors & \multicolumn{3}{|c|}{ Constant } & \multicolumn{3}{c|}{ Random } \\
\hline$\Delta[\mathrm{nT}]$ & $X[\mathrm{nT}]$ & $Y[\mathrm{nT}]$ & $Z[\mathrm{nT}]$ & $X[\mathrm{nT}]$ & $Y[\mathrm{nT}]$ & $Z[\mathrm{nT}]$ \\
\hline 0.004 & 0.04 & 0.04 & 0.01 & 0.04 & 0.04 & 0.01 \\
0.02 & 0.21 & 0.22 & 0.06 & 0.20 & 0.21 & 0.06 \\
0.1 & 1.04 & 1.09 & 0.32 & 1.00 & 1.05 & 0.32 \\
0.5 & 5.20 & 5.4 & 1.6 & 5.0 & 5.2 & 1.6 \\
2.5 & 26.0 & 27.2 & 8.1 & 24.9 & 26.2 & 7.9 \\
\hline
\end{tabular}


Table $3 b$

The mean absolute errors of $X, Y$, and $Z$ components due to the total field variation during measurement cycle (with sequence times 1 to $4 \mathrm{~s}$ ), for four observatories (RES, IQA, STJ, and KOU) during quiet and disturbed conditions, respectively

\begin{tabular}{|c|c|c|c|c|c|c|}
\hline Errors [nT] & \multicolumn{3}{|c|}{ Quiet } & \multicolumn{3}{|c|}{ Disturbed } \\
\hline$\tau / s$ & $X$ & $Y$ & $Z$ & $X$ & $Y$ & $Z$ \\
\hline \multicolumn{7}{|c|}{ RES } \\
\hline 1 & 0.12 & 0.12 & 0.005 & 0.23 & 0.39 & 0.013 \\
\hline 2 & 0.12 & 0.22 & 0.007 & 0.48 & 0.68 & 0.026 \\
\hline 3 & 0.14 & 0.24 & 0.007 & 0.65 & 0.98 & 0.036 \\
\hline 4 & 0.18 & 0.21 & 0.009 & 0.81 & 1.29 & 0.046 \\
\hline 0.2 & 0.010 & 0.014 & 0.001 & 0.043 & 0.066 & 0.002 \\
\hline \multicolumn{7}{|c|}{ IQA } \\
\hline 1 & 0.08 & 0.14 & 0.02 & 0.54 & 1.39 & 0.17 \\
\hline 2 & 0.13 & 0.26 & 0.03 & 1.14 & 2.73 & 0.34 \\
\hline 3 & 0.18 & 0.38 & 0.05 & 1.79 & 4.02 & 0.50 \\
\hline 4 & 0.23 & 0.49 & 0.06 & 2.54 & 5.25 & 0.67 \\
\hline 0.2 & 0.012 & 0.025 & 0.003 & 0.12 & 0.27 & 0.034 \\
\hline \multicolumn{7}{|c|}{ STJ } \\
\hline 1 & 0.32 & 0.34 & 0.13 & 0.41 & 0.48 & 0.18 \\
\hline 2 & 0.32 & 0.35 & 0.13 & 0.52 & 0.66 & 0.24 \\
\hline 3 & 0.37 & 0.36 & 0.15 & 0.68 & 0.86 & 0.33 \\
\hline 4 & 0.36 & 0.39 & 0.15 & 0.84 & 1.07 & 0.40 \\
\hline 0.2 & 0.023 & 0.025 & 0.010 & 0.046 & 0.058 & 0.022 \\
\hline \multicolumn{7}{|c|}{$\mathrm{KOU}$} \\
\hline 1 & 0.03 & 0.08 & 0.06 & 0.03 & 0.08 & 0.07 \\
\hline 2 & 0.05 & 0.09 & 0.11 & 0.06 & 0.15 & 0.11 \\
\hline 3 & 0.06 & 0.13 & 0.11 & 0.05 & 0.13 & 0.16 \\
\hline 4 & 0.04 & 0.10 & 0.10 & 0.05 & 0.15 & 0.17 \\
\hline 0.2 & 0.003 & 0.007 & 0.007 & 0.003 & 0.009 & 0.010 \\
\hline
\end{tabular}

Note: The estimated errors for $\tau=0.2 \mathrm{~s}$ are derived from errors for sequence times 1 to $4 \mathrm{~s}$.

to the ocean coast effect) and smallest for KOU. They are then largest in $Y$ and smallest in $Z$ component for RES, IQA, and STJ, while for KOU are largest in $Y$ and smallest in $X$. They also increase with sequence time, but not so obvious as for disturbed conditions (except for IQA). The very rough errors estimates for $\tau=0.2 \mathrm{~s}$ are based on the errors for sequence times 1 to $4 \mathrm{~s}$ (linear dependence of errors on sequence times). 


\subsection{The instrumental accuracy}

To the noise level of dIdD, beside a thermal noise of sensor and a noise of electronic circuits connected to the sensor, there contributes a noise from the bias fields' current source. They produce additional fluctuations in measured components $D_{p}, D_{m}, I_{p}$, and $I_{m}$. For their root-mean-square (rms) noise levels estimation, a single instrument analysis was used, and it works well when the geomagnetic field is in a quiet, undisturbed conditions. The first differences between successive one-second data of particular component were computed, and then the root-mean-square errors of successive five-minute moving averages were derived. The minimal values of these errors during quiet intervals were the corresponding noise levels (Worthington et al. 2009). This method was approved by applying it to the data derived by adding a white noise of known amplitudes on quiet time variations (from SQ1 Model), and it was found that this gives quite reasonable estimates of noise levels (noise amplitudes). In Table 4 the estimates for measured components in Milton are given.

Table 4

The estimated rms noise levels of measured components, for P-dIdD in Milton

\begin{tabular}{|c|c|c|c|c|c|c|}
\hline rms noise level & $\begin{array}{c}M_{D p} \\
{[\mathrm{pT}]}\end{array}$ & $\begin{array}{c}M_{D m} \\
{[\mathrm{pT}]}\end{array}$ & $\begin{array}{c}M_{F} \\
{[\mathrm{pT}]}\end{array}$ & $\begin{array}{c}M_{I p} \\
{[\mathrm{pT}]}\end{array}$ & $\begin{array}{c}M_{I m} \\
{[\mathrm{pT}]}\end{array}$ & $\begin{array}{c}\text { Mean } \\
{[\mathrm{pT}]}\end{array}$ \\
\hline P-dIdD & 9 & 9 & 6 & 9 & 9 & $8 \pm 1$ \\
\hline
\end{tabular}

Note: These values are the mean over five days, when $K_{\text {Отт }} \leq 1$.

By using the data from Table 4 one can estimate the maximal (shortterm) theoretical accuracies of calculated components, and they were for $X$, $Y, H$ (horizontal field), and $Z$, respectively: $56,59,56$, and $21 \mathrm{pT}$. The mean field values were taken as the averages of geomagnetic components over five days. One can also estimate that the bias fields accuracies for Milton data are $\pm 0.08 \mathrm{nT}$. The relative error of assumption that both bias fields are equal is about $0.09 \%$, and in this case the mean bias field is $5853 \mathrm{nT}$ ( $5 \mathrm{~mA}$ bias current).

Additionally, the Overhauser effect dIdD was operated at $1 \mathrm{~Hz}$ sampling rate with $11960 \mathrm{nT}$ mean bias field, in the interval 16-20 May 2012, on the nonmagnetic pillar some $20 \mathrm{~m}$ away from P-dIdD pillar, due to the similar reasons as P-dIdD. The mean estimated rms noise level of measured components was $144 \pm 9 \mathrm{pT}$, and the estimated maximal theoretical accuracies of $X$, $Y$, and $Z$ were 426,484 , and $195 \mathrm{pT}$, respectively.

The next figure shows the maximal theoretical accuracies of calculated components as functions of the bias fields, when $A_{\mathrm{i}}=A_{\mathrm{d}}=4000-8000 \mathrm{nT}$. 
The shown results are the estimations for RES, IQA, STJ, and KOU. The values for geomagnetic elements were derived from IGRF-11 Model (http:// www.geomag.us/online calculators.html) on the middle of May 2012. The calibration parameter values were: $D_{0}=D_{\mathrm{IGRF}}, I_{0}=I_{\mathrm{IGRF}}, \quad \theta=0.5^{\circ}$ and $\varepsilon_{O}=0.1^{\circ}$. From Fig. 5 one can notice that the estimated maximal theoretical accuracies increase with increasing bias fields, and depend on geomagnetic coordinates. The $X$ and $Y$ components accuracies increase with decreasing geomagnetic latitude, i.e., both are higher in equatorial than in polar cap region, and for the same bias field are smaller for $Y$ component. In the case of
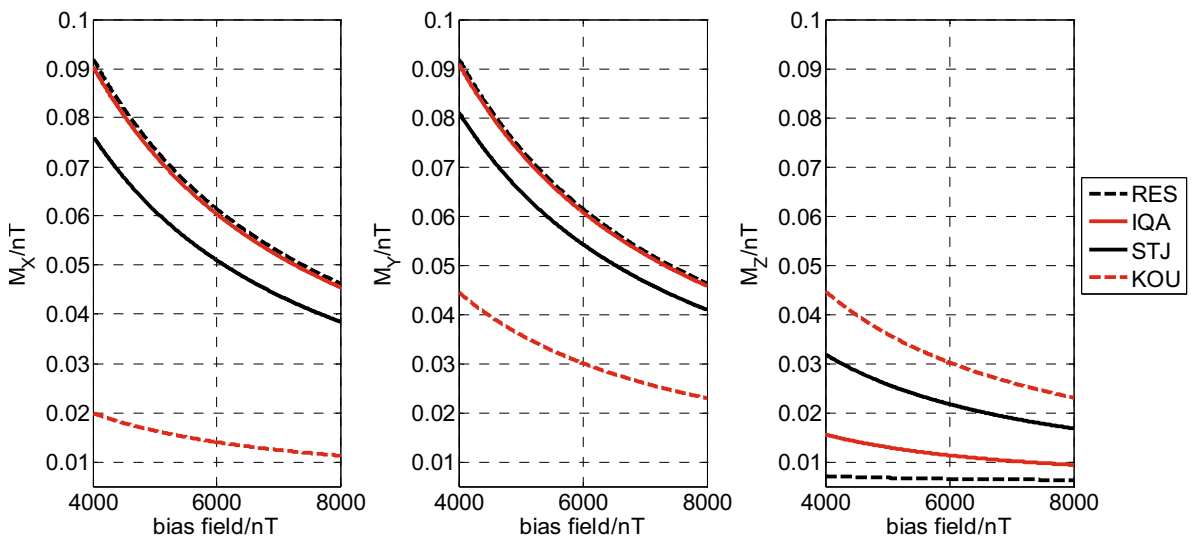

Fig. 5. The estimated maximal theoretical accuracies of directional components for the four observatories (RES, IQA, STJ, and KOU), as the functions of bias field, for May 2012.
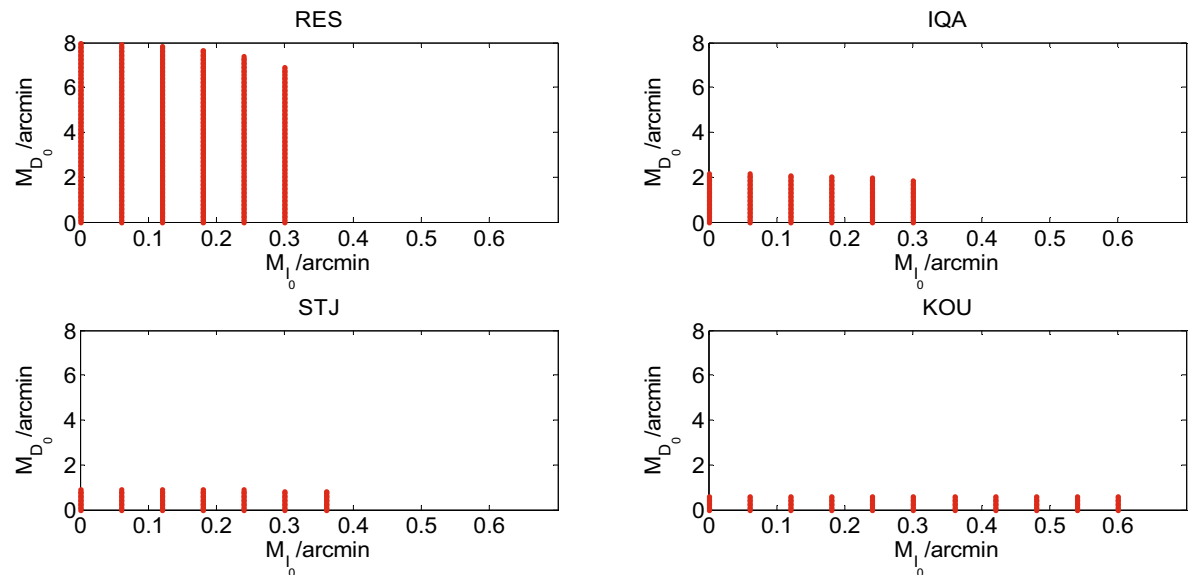

Fig. 6. The long-term $D_{0}$ and $I_{0}$ accuracies, i.e., $\left(M_{I 0}, M_{D 0}\right)$ pairs, when $M_{\theta}=0.5^{\circ}$ and $M_{\varepsilon o}=0.2^{\circ}$, needed to achieve the $X, Y$, and $Z$ accuracies higher than $5 \mathrm{nT}$, for RES, IQA, STJ, and KOU observatories, respectively. 
the $Z$ component accuracies, they are highest in the polar cap region (almost constant with respect to the bias field) and smallest in the equatorial region.

The long term accuracies will depend, beside on measured components' accuracies, on long-term accuracies of $D_{0}, I_{0}, \theta$, and $\varepsilon_{O}$. These parameters could be time-dependent during humidity and temperature uncontroled conditions. The long-term accuracies estimation was made under the same assumptions about the mean components and calibration parameters values, as the maximal accuracies. By using some characteristic one-year accuracies under controlled conditions (Heilig 2007), it is possible to estimate the longterm accuracies. In this case study, the values of $M_{\theta}=0.5^{\circ}$ and $M_{\varepsilon o}=0.2^{\circ}$ were taken, and the pairs of $\left(M_{I 0}, M_{D 0}\right)$ values were found, so that the longterm accuracies of $X, Y$, and $Z$ were higher than $5 \mathrm{nT}$. The results for RES, IQA, STJ, and KOU are shown in Fig. 6, where each dot represents $\left(M_{I 0}\right.$, $\left.M_{D 0}\right)$ pair.

\section{CONCLUSIONS}

During the presented short-term tests, dIdD has operated with $1 \mathrm{~Hz}$ sampling rate during five days on a stable nonmagnetic pillar. After instrument setup and calibration, attention was focused on stability tests, considering the bias fields and the instrumental noise level. It was estimated that the D- and I-coils bias fields are quite stable when considering their current source fluctuations, and confirmed that there is a negative correlation between the bias fields and the surrounding air temperature. One can presume that this is due to the small temperature changes of bias coils dimensions. The calculated components' correlations with the nearest observatory (Ottawa) were high.

The errors due to the instrument orientation assumptions (with deviations up to $0.1^{\circ}$ ) were estimated to be the most sensitive on the calibration parameter $I_{0}$ in the case of $X, Y$, and $Z$ components for polar (up to $87 \mathrm{nT}$ ) and auroral observatory (up to $85 \mathrm{nT}$ ); and $X$ and $Z$ components for mid-latitude and equatorial observatory (up to 80 and $49 \mathrm{nT}$, respectively). Parameter $D_{0}$ had the highest influence on $Y$ component at mid-latitude and equatorial observatory (up to 33 and $47 \mathrm{nT}$, respectively). The parameters $\theta$ and $\varepsilon_{O}$ had very small influence, but the errors due to them were relatively severely increased during disturbed conditions (up to 8 and $5 \mathrm{nT}$ for $Y$ component in auroral region, respectively). The orientation errors in Milton were less than $1 \mathrm{nT}$. The errors due to the total field time variations during dIdD measurement cycle, based on synthetic and real data from four observatories and Milton, were estimated for different cycle times and during different geomagnetic conditions. It was estimated that these errors are generally larger for longer cycle time and during disturbed conditions (up to $5 \mathrm{nT}$ for $Y$ component in auroral region at $20 \mathrm{~s}$ cycle time). 
On the basis of measured components' noise level (better than $10 \mathrm{pT}$ in rms sense) one can estimate the geomagnetic components maximal theoretical accuracies, and they depend also on the position of measurement site on the Earth. In this case study it was estimated that the highest short-term accuracies for horizontal components will be in equatorial regions (better than $20 \mathrm{pT}$ in $X$ and $40 \mathrm{pT}$ in $Y$ ), and for vertical component in polar region (better than $10 \mathrm{pT}$ ). Also it was estimated that the long-term geomagnetic components' accuracies will depend dominantly on $D_{0}$ and $I_{0}$ accuracies, with lowest $D_{0}$ and highest $I_{0}$ accuracies in polar region, and vice versa in equatorial regions.

The mechanical design of P-dIdD is the same as for the Overhauser effect dIdD, but at the same sampling rate of $1 \mathrm{~Hz}$ the P-dIdD has about 18 times better measured components' accuracy, and about 7-9 times better the maximal theoretical accuracies of $X, Y$, and $Z$ components (at mid-latitudes). All the results in this study considering Potassium dIdD at $1 \mathrm{~Hz}$ sampling rate could indicate that it can be suitable for, e.g., the geomagnetic observatories, repeat station, directional drilling surveys, and could be also used in some ionospheric or magnetospheric studies.

Acknowledgments. The author is deeply grateful to Dr. I. Hrvoic for providing him training at GEM System Inc., and for providing Potassium dIdD for this study. The staff of GEM System Inc. participating in the field work are kindly acknowledged. The author thanks the national institutes that support geomagnetic observatories and Intermagnet for promoting high standards of magnetic observatory practice (www.intermagnet.org). The Ottawa Geomagnetic Observatory and the Weather Station Guelph Turfgrass are kindly acknowledged for providing the data. The author would like to thank Dr. M. Brkić (Zagreb) and M.Sc. B. Heilig (Tihany) for valuable comments on the manuscript. The Associate Editor Dr. M. Zarroca and two anonymous reviewers are acknowledged for their constructive comments that improved the manuscript.

References

Alexandrov, E.B., and V.A. Bonch-Bruevich (1992), Optically pumped atomic magnetometers after three decades, Opt. Eng. 31, 4, 711-717, DOI: $10.1117 / 12.56132$.

Alexandrov, E.B., M.V. Balabas, V.N. Kulyasov, A.E. Ivanov, A.S. Pazgalev, J.L. Rasson, A.K. Vershovski, and N.N. Yakobson (2004), Three-compo- 
nent variometer based on a scalar potassium sensor, Meas. Sci. Technol. 15, 5, 918-922, DOI: 10.1088/0957-0233/15/5/020.

Alldredge, L.R. (1960), A proposed automatic standard magnetic observatory, J. Geophys. Res. 65, 11, 3777-3786, DOI: 10.1029/JZ065i011p03777.

Alldredge, L.R., and I. Saldukas (1964), An automatic standard magnetic observatory, J. Geophys. Res. 69, 10, 1963-1970, DOI: 10.1029/JZ069i010p01963.

Auster, H.U., and V. Auster (2003), A new method for performing an absolute measurement of the geomagnetic field, Meas. Sci. Technol. 14, 7, 10131017, DOI: 10.1088/0957-0233/14/7/315.

Auster, H.U., M. Mandea, A. Hemshorn, E. Pulz, and M. Korte (2007), Automation of absolute measurement of the geomagnetic field, Earth Planets Space 59, 9, 1007-1014, DOI: 10.1186/BF03352041.

Auster, V., O. Hillenmaier, R. Kroth, and M. Wiedemann (2007), Advanced proton magnetometer design and its application for absolute measurement, Publs. Inst. Geophys. Pol. Acad. Sc. C-99, 398, 60-68.

Bell, W.E., and A.L. Bloom (1957), Optical detection of magnetic resonance in alkali metal vapor, Phys. Rev. 107, 6, 1559-1565, DOI: 10.1103/PhysRev. 107.1559 .

Campbell, W.H. (2003), Introduction to Geomagnetic Fields, 2nd ed., Cambridge University Press, Cambridge.

Csontos, A., L. Hegymegi, and B. Heilig (2007), Temperature tests on modern magnetometers, Publs. Inst. Geophys. Pol. Acad. Sc. C-99, 398, 171-177.

Csontos, A., D. Šugar, M. Brkić, P. Kovács, and L. Hegymegi (2012), How to control a temporary DIDD based observatory in the field? Ann. Geophys. 55, 6, 1085-1094, DOI: 10.4401/ag-5447.

Dehmelt, H.G. (1957), Modulation of a light beam by precessing absorbing atoms, Phys. Rev. 105, 6, 1924-1925, DOI: 10.1103/PhysRev.105.1924.

Gu, Z., Z. Zhan, J. Gao, T. Yao, and W. Han (2006), Application of GPS in geomagnetic survey, Earth Planets Space 58, 6, 723-729, DOI: 10.1186/ BF03351974.

Happer, W. (1972), Optical pumping, Rev. Mod. Phys. 44, 2, 169-249, DOI: 10.1103/RevModPhys.44.169.

Hegymegi, L., B. Heilig, and A. Csontos (2004), New suspended dIdD magnetometer for observatory (and field?) use. In: Proc. 11th IAGA Workshop on Geomagnetic Observatory Instruments, Data Acquisition and Processing, 9-17 November 2004, Kakioka and Tsukuba, Japan, 28-33.

Heilig, B. (2007), Intercalibration of dIdD and Fluxgate Magnetometers, Publs. Inst. Geophys. Pol. Acad. Sc. C-99, 398, 144-151.

Hrvoic, I., and L.R. Newitt (2011), Instruments and methodologies for measurement of the Earth's magnetic field. In: M. Mandea and M. Korte (eds.), Geomagnetic Observations and Models, IAGA Special Sopron Book Series, 
Vol. 5, Springer-Verlag, Berlin Heidelberg, 105-126, DOI: 10.1007/97890-481-9858-0 5.

Hrvoic, I., G. Cifuentes-Nava, E. Cabral-Cano, E. Hernandez, M. Wilson, and F. Lopez (2009), New magnetic observatory installation in Oaxaca, Mexico. In: Proc. 13th IAGA Workshop on Geomagnetic Observatory Instruments, Data Acquisition and Processing, 9-18 June 2008, Boulder and Golden, CO, USA.

Jankowski, J., and C. Sucksdorff (1996), Guide for Magnetic Measurements and Observatory Practice, International Association of Geomagnetism and Aeronomy, Warszawa, Poland, $235 \mathrm{pp}$.

Marsal, S., J.M. Torta, and J.C. Riddick (2007), An assessment of the BGS $\delta \mathrm{D} \delta \mathrm{I}$ vector magnetometer, Publs. Inst. Geophys. Pol. Acad. Sc. C-99, 398, 158165.

Newitt, L.R., C.E. Barton, and J. Bitterly (1996), Guide for Magnetic Repeat Station Surveys, International Association of Geomagnetism and Aeronomy, Warszawa, Poland.

Pang, H., J. Li, D. Chen, M. Pan, S. Luo, Q. Zhang, and F. Luo (2013), Calibration of three-axis fluxgate magnetometers with nonlinear least square method, Measurement 46, 4, 1600-1606, DOI: 10.1016/j.measurement.2012.11.001.

Schott, J.J., and P. Leroy (2001), Orientation of the DIDD magnetometer, Contrib. Geophys. Geod. 31, 1, 43-50.

Schott, J.J., V. Boulard, A. Pérès, and J.M. Cantin (2001), Magnetic component measurements with the DIDD, Contrib. Geophys. Geod. 31, 1, 35-42.

St-Louis, B. (ed.), (2004), Intermagnet technical reference manual: Version 4.2, http://www.intermagnet.org.

van Loo, S.A., and J.L. Rasson (2007), Presentation of the prototype of an automated DIFlux, Publs. Inst. Geophys. Pol. Acad. Sc. C-99, 398, 77-86.

Worthington, E.W., E.A. Sauter, and J.J. Love (2009), Analysis of USGS onesecond data. In: Proc. 13th IAGA Workshop on Geomagnetic Observatory Instruments, Data Acquisition and Processing, 9-18 June 2008, Boulder and Golden, CO, USA. 